\title{
Article
}

\section{Physiological, kinematic, and electromyographic responses to kinesiology-type patella tape in elite cyclists}

Hébert-Losier, K, Yin, NS, Beavena, CM, Li, CTC and Richards, James

Available at http://clok.uclan.ac.uk/24887/

Hébert-Losier, K, Yin, NS, Beavena, CM, Li, CTC and Richards, James ORCID: 0000-0002-4004-3115 (2019) Physiological, kinematic, and

electromyographic responses to kinesiology-type patella tape in elite cyclists. Journal of Electromyography and Kinesiology, 44 . pp. 36-45. ISSN 1050-6411

It is advisable to refer to the publisher's version if you intend to cite from the work. http://dx.doi.org/10.1016/j.jelekin.2018.11.009

For more information about UCLan's research in this area go to http://www.uclan.ac.uk/researchgroups/ and search for <name of research Group>.

For information about Research generally at UCLan please go to http://www.uclan.ac.uk/research/

All outputs in CLoK are protected by Intellectual Property Rights law, including Copyright law. Copyright, IPR and Moral Rights for the works on this site are retained by the individual authors and/or other copyright owners. Terms and conditions for use of this material are defined in the policies page. 
1 Physiological, kinematic, and electromyographic responses to kinesiology-type patella

2 tape in elite cyclists

3

4 Kim Hébert-Losier ${ }^{\mathrm{a} *}$, Ngieng Siew Yin ${ }^{\mathrm{b}}$, C. Martyn Beaven ${ }^{\mathrm{a}}$, Chris Tee Chow Li ${ }^{\mathrm{b}}$, and Jim

5 Richards ${ }^{\mathrm{c}}$

6

$7 \quad{ }^{a}$ Faculty of Health, Sport and Human Performance, University of Waikato, Tauranga, New

$8 \quad$ Zealand

9

$10{ }^{b}$ Department of Sports Science, National Sports Institute of Malaysia, Kuala Lumpur,

11 Malaysia

12

$13{ }^{b}$ School of Health Sciences, University of Central Lancashire, Preston, Lancashire, United

$14 \quad$ Kingdom

15

16 *Corresponding Author: Kim Hébert-Losier,

17 e-mail: kim.hebert-losier@waikato.ac.nz

18

19 Keywords: 3D analysis; biomechanics; electromyography; kinesiology; performance;

20 physiology

21

22 CONFLICT OF INTEREST

23 None.

24

25 FINANCIAL DISCLOSURE

26 None.

27 


\section{ABSTRACT}

30 Kinesiology-type tape (KTT) has become popular in sports for injury prevention,

31 rehabilitation, and performance enhancement. Many cyclists use patella KTT; however, its

32 benefits remain unclear, especially in uninjured elite cyclists. We used an integrated

33 approach to investigate acute physiological, kinematic, and electromyographic responses to

34 patella KTT in twelve national-level male cyclists. Cyclists completed four, 4-minute

35 submaximal efforts on an ergometer at 100 and $200 \mathrm{~W}$ with and without patella KTT.

36 Economy, energy cost, oxygen cost, heart rate, efficiency, 3D kinematics, and lower-body

37 electromyography signals were collected over the last minute of each effort. Comfort levels

38 and perceived change in knee stability and performance with KTT were recorded.

39 The effects of KTT were either unclear, non-significant, or clearly trivial on all collected

40 physiological and kinematic measures. KTT significantly, clearly, and meaningfully

41 enhanced vastus medialis peak, mean, and integrated electromyographic signals, and vastus

42 medialis-to-lateralis activation. Electromyographic measures from biceps femoris and

43 biceps-to-rectus femoris activation ratio decreased in either a significant or clinically

44 meaningful manner. Despite most cyclists perceiving KTT as comfortable, increasing

45 stability, and improving performance, the intervention exerted no considerable effects on all

46 physiological and kinematic measures. KTT did alter neuromuscular recruitment, which has

47 potential implications for injury prevention. 
49 Many health professionals, athletes, and coaches use kinesiology-type tape (KTT), with the

50 intent to manage musculoskeletal sport injuries; however, growing evidence suggests no

51 additional benefit from KTT application compared to placebo taping or active control

52 treatment methods when managing musculoskeletal conditions (Ouyang et al., 2018,

53 Williams et al., 2012). On the other hand, several beneficial effects from KTT application

54 have been reported, including enhancement in muscle activation (Gilleard et al., 1998);

55 improved biomechanics, joint, and patella alignment (Lyman et al., 2017, Merino-Marban et

56 al., 2013); and decreased pain (Bockrath et al., 1993, Merino-Marban et al., 2013). It is

57 worth noting, however, that many studies report no effect from KTT on these measures

58 (Halski et al., 2015) or athletic performance (Lins et al., 2013, Reneker et al., 2018).

59 Underlying reasons for such contrasting scientific findings likely include the varied

60 application methods, differences in the mechanical properties of KTT across brands

61 (Matheus et al., 2017), targeted population, and individuals' perceived benefits of KTT with

62 a potential for placebo effect (Mak et al., 2018) .

63

64 Cycling is a popular recreational and competitive sporting activity worldwide, and a

65 common exercise modality used during rehabilitation. At an elite level, athletes and coaches

66 continually seek for ways to improve performance through marginal gains and prevent the

67 occurrence of injuries. KTT is routinely used by coaches and athletes as an ergogenic aid

68 (Reneker et al., 2018), with various forms of taping employed to prevent injury occurrence

69 or recurrence (Zech and Wellmann, 2017). Given that up to $94 \%$ of professional cyclists

70 suffer from at least one overuse injury annually (Silberman, 2013), the visible increase in

71 use of KTT amongst elite cyclists for prophylactic purposes is not surprising.

72

73 Taping or bracing are frequently used to alleviate patellofemoral pain (PFP) symptoms and

74 can impact knee motion during cycling (Theobald et al., 2012). Non-specific KTT

75 application has been shown to be as effective as specific application for reducing pain and 
inducing significant changes in lower-body cycling biomechanics in a symptomatic

77 population group (Theobald et al., 2014). However, any potential positive effect of taping on

78 energy cost of cycling, neuromuscular recruitment patterns, and performance of

79 asymptomatic high-level cyclists has not been examined, despite the visibly increased

80 prevalence of use in the cycling community and KTT marketing campaigns.

82 Using an integrated approach, our aim was therefore to investigate the acute physiological,

83 kinematic, and electromyographic outcomes in response to applying KTT to the knee of elite

84 cyclists. As perceptions may influence outcomes of interventions, individual perceptions

85 were also assessed. We hypothesised that taping would be accompanied by changes in

86 muscle recruitment patterns, cycling economy and efficiency, and perceived stability that

87 have the potential to modulate cycling performance.

\section{MATERIALS \& METHODS}

\section{$90 \quad$ Participants}

91 All male cyclists of the National Cycling Team training at the National Sports Institute for 92 Malaysia $(n=12)$ were invited and accepted to participate in this study. These 12 national 93 cyclists (mean \pm standard deviation (SD): age, $21.7 \pm 2.8$ years; body mass, $65.6 \pm 5.4 \mathrm{~kg}$; 94 and height, $172.7 \pm 3.4 \mathrm{~cm}$ ) with at least four years of training experience provided written 95 informed consent to participate in this study, which adhered to The Code of Ethics of the 96 World Medical Association (Declaration of Helsinki) and was approved by our Institutional

97 Ethical Review Board (ISNRP 29/2015). Inclusion criteria were cyclists training at the 98 National Sports Institute of Malaysia for the National Cycling Team, good self-reported 99 general health, and at least 18 years of age. Cyclists with current or recent $(<1$ month $)$ musculoskeletal injuries, joint pathologies, or medical contraindications to physical exertion were excluded. 
104 All cyclists attended 3 sessions at the biomechanics laboratory of the National Sports

105 Institute of Malaysia, one week apart. The first two weeks were familiarization sessions and

106 the third week was used to investigate the acute effects of KTT application through a

107 repeated-measures randomized experimental study design.

109 Given that ergometer versus outdoor cycling can affect cycling physiological measures

110 (Bertucci et al., 2012) and pedalling biomechanics (Bertucci et al., 2007), cyclists brought

111 their road bikes to the laboratory the first week of testing. Bike setup parameters were

112 recorded and employed to individualize setup on a Lode cycling ergometer (Excalibur Sport,

113 Lode B.V., Groningen, Netherlands), with all cyclists using their habitual cleats. The final

114 ergometer setup was recorded and used across the three weeks. Baseline demographics,

115 including leg dominance (self-perceived stronger cycling side), were recorded. Body mass

$116(\mathrm{~kg})$ was measured weekly and subsequently used to calculate relative physiological

117 variables.

119 Cyclists began all sessions with a 2-min cycling warm-up on the ergometer after being set-

120 up with the monitoring equipment. Cyclists then performed a submaximal 4-minute cycling

121 effort at $100 \mathrm{~W}$, followed immediately by a second submaximal 4-minute cycling effort at

$122200 \mathrm{~W}$. The efforts were completed without KTT during the familiarization weeks, and

123 twice on the third week: once with and once without KTT. The no tape (NT) and KTT

124 conditions were completed in a block-randomized order and separated by a 5-minute passive

125 seated rest. Thus, all cyclists completed four 4-minute efforts: NT $100 \mathrm{~W}$, NT $200 \mathrm{~W}, \mathrm{KTT}$

$126100 \mathrm{~W}$, and KTT $200 \mathrm{~W}$. The powers of 100 and $200 \mathrm{~W}$ were selected to ensure that cycling

127 efforts were below the anaerobic threshold and to compute delta efficiency (Coyle et al.,

128 1992) (see Physiology). Furthermore, the application of patella KTT at these powers has

129 been shown to alter lower-body cycling biomechanics in previous studies (Theobald et al.,

130 2014), with these power levels set alongside the National Cycling Team of Malaysia to

131 inform their practice and use of KTT application in longer steady-state riding situations. 


\section{Taping method}

134 The taping application we used was based on a method previously reported to induce

135 changes in cycling biomechanics within the power ranges here examined (Theobald et al.,

136 2012, Theobald et al., 2014). With cyclists seated on the ergometer with the leg at the

137 bottom of their power stroke, a strip of KTT (RockTape ${ }^{\mathrm{TM}}$, RockTape Inc., California) of

138 length equal to $50 \%$ of individual knee circumference was applied on to the centre of the

139 patella with light tension (approximately $25 \%$ of stretch to the tape). The medial and lateral

140 tape edges were aligned with the medial and lateral knee-joint lines (Figure 1). The same

141 experienced physiotherapist applied the tape to all cyclists. This simple KTT method (i.e.,

142 across the patella) was selected given its ease-of-use and findings from previous studies

143 indicating that such a method impacts cycling biomechanics in a manner that is comparable

144 to that of a more intricate KTT application method (Theobald et al., 2014). Given the

145 minimalist KTT method applied, a "placebo" taping method was not implemented.

$146 * * *$ Insert Figure 1***

\section{Physiology}

149 Oxygen consumption $\left(\mathrm{VO}_{2}\right)$, carbon dioxide output $\left(\mathrm{VCO}_{2}\right)$, and heart rate were monitored 150 throughout the 4-minute experimental efforts using a calibrated K5 wearable metabolic technology system (COSMED, Rome, Italy). All physiological measures were averaged over the last minute where steady state was observed. $\mathrm{VO}_{2}$ was used to determine steady-state

153 relative oxygen cost $(\mathrm{mL} / \mathrm{kg} / \mathrm{km})$ and absolute cycling economy $(\mathrm{W} / \mathrm{L} / \mathrm{min})$. From the $\mathrm{VO}_{2}$

154 and $\mathrm{CO}_{2}$ data, the relative energy cost of efforts $(\mathrm{kcal} / \mathrm{kg} / \mathrm{km})$ was estimated using the energy 155 expenditure equations described by Jeukendrup and Wallis (2005). Gross efficiency (\%) and 156 delta efficiency (\%) were calculated as suggested by Coyle et al. (1992) using the ratio of 157 work accomplished (watts converted to $\mathrm{kcal} / \mathrm{min}$ ) to absolute energy cost ( $\mathrm{kcal} / \mathrm{min}$ ) for 158 gross efficiency, and the reciprocal of the slope that describes the relationship between the 159 absolute energy cost and work accomplished for delta efficiency. 


\section{$161 \quad$ Kinematics}

162 Lower-body, trunk, and pelvis movements were captured in 3D during the last minute of 163 each 4-minute cycling effort at $300 \mathrm{~Hz}$ using 10 Oqus 300 infrared cameras and the Qualisys

164 Track Manager Software version 2.12 (Qualisys AB, Gothenburg, Sweden). Forty-six retroreflective markers (12 $\mathrm{mm}$ in diameter) were affixed to the skin, clothes, and shoes of cyclists based on the Calibrated Anatomical System Technique (Cappozzo et al., 1997) and

167 following established guidelines (Grood and Suntay, 1983). All 46 markers were used for 168 static calibration; whereas 14 markers were removed for the cycling efforts (Figure 2).

172 An 8-segment biomechanical model with 6 degrees of freedom at each joint was constructed 173 in Visual3D Professional ${ }^{\mathrm{TM}}$ Software version 5.02.30 (C-Motion Inc., Germantown, MD, 174 USA), with the local coordinates of the trunk, pelvis, thighs, shanks, and feet derived from 175 the static calibration and the pelvis used to define hip-joint centres (Bell et al., 1989). Prior 176 to each session, the measurement volume was calibrated using a 750-mm wand and L-frame 177 that defined the Cartesian origin of the laboratory. Cyclists were then requested to sit on the 178 saddle of the ergometer, with legs hanging to the side, and remain motionless to allow static 179 calibration.

\section{Electromyography}

182 The electromyography (EMG) signals from the following four muscles were recorded on 183 both the dominant and non-dominant sides: vastus medialis (VM), vastus lateralis (VL), rectus femoris $(\mathrm{RF})$ and biceps femoris $(\mathrm{BF})$. Signals were recorded using Noraxon's Dual

185 EMG surface $\mathrm{Ag} / \mathrm{AgCl}$ electrodes (17.5 mm inter-electrode distance), wireless EMG sensors, and Desktop DTS data logger (Noraxon USA Inc., Scottsdale, AZ). EMG data were sampled at $1500 \mathrm{~Hz}$, low-pass filtered at $500 \mathrm{~Hz}$, and digitally integrated through the 
Qualisys Track Manager Software. Skin preparation and electrode positioning followed the

189 Surface EMG for Noninvasive Assessment of Muscle (Hermens et al., 2000), International

190 Society of Electrophysiology and Kinesiology (Merletti and di Torino, 1999), and published

191 protocols (Gilleard et al., 1998). Cyclists completed a few cycling revolutions before

192 experimentation to allow visual inspection of EMG signal quality. Sensors were checked and 193 reapplied if artefacts were observed.

\section{Perception}

196 Perceived change in knee stability and performance with KTT compared to NT was assessed

197 at the end of the experimental session using a 5-point Likert (1932) Scale from negative (1)

198 to positive (5) perception, with the mid-point value representing no change (3). Comfort

199 level of KTT was also assessed using a similar method. Anchor points ranged from very

200 uncomfortable (1), much less stable (1), and much worse (1) to very comfortable (5), much

201 more stable (5), and much better (5) for comfort, knee stability, and performance,

202 respectively.

\section{Data processing}

205 Kinematic and EMG data were exported to the C3D format and processed in Visual 3D.

206 Marker data were filtered using a $4^{\text {th }}$ order zero-lag $15 \mathrm{~Hz}$ Butterworth bidirectional filter.

207 Kinematic parameters were then calculated using rigid-body analysis and Euler angles

208 obtained from the static calibration. Hip, knee, and ankle angles in the sagittal (flexion-

209 extension), coronal (adduction-abduction), and transverse (internal-external rotation) planes

210 were calculated using an $x-y-z$ Cardan sequence equivalent to the Joint Coordinate System

211 (Grood and Suntay, 1983), with the pelvis angles in the sagittal (anterior-posterior), coronal

212 (dominant, non-dominant obliquity), and transverse (dominant, non-dominant rotation)

213 planes defined relative to the laboratory. Trunk angles in the sagittal (flexion-extension),

214 coronal (dominant, non-dominant lateral flexion), and transverse (dominant, non-dominant

215 rotation) planes were also defined in relation to the laboratory coordinates. Data were 
216 divided into movement cycles and time-normalized based on maximal knee flexion events.

217 Ensemble-average kinematic curves were generated for each participant and cycling effort,

218 and range of motion (ROM) values extracted.

220 EMG signal data were zeroed to remove any baseline offset and a 20-Hz high-pass filter

221 applied to remove movement artefacts. Signals were subsequently rectified and linear

222 envelopes generated by smoothing the data using a low-pass, $4^{\text {th }}$ order, zero-lag $15 \mathrm{~Hz}$

223 Butterworth filter. The linear envelope for each muscle was then normalized to the highest

224 observed signal across all four conditions examined (\% max). Similar to the kinematic data,

225 ensemble-average EMG signal curves time normalized to maximal knee flexion events were

226 generated from which mean and peak EMG signal values were extracted. An integrated

227 EMG (iEMG) signal was also generated by integrating the linear envelop from the start to

228 the end of each movement cycle, which was then normalized to the maximal observed iEMG

229 across all four efforts (\% max).

231 Statistical analysis

232 Mean and SD values were computed for all parameters for both the 100 and $200 \mathrm{~W}$ efforts

233 and dominant and non-dominant sides. Changes in mean $\left(\Delta_{\text {mean }}\right)$ and standardized effect

234 sizes (ES) were computed to quantify the acute effect of KTT; with ES considered small,

235 moderate, large, and very large when reaching thresholds of $0.2,0.6,1.2,2.0$, and trivial

236 when < 0.2 (Smith and Hopkins, 2011). An effect was deemed 'clear' when its 90\%

237 confidence limit did not overlap the thresholds for small positive and small negative effects

238 (i.e., 5\%); and 'likely' to be clinically meaningful when its probability exceeded $75 \%$ (Smith

239 and Hopkins, 2011).

240

241 Paired $t$-tests were used to investigate differences between the tape and no-tape condition for

242 the measures of interest, with the threshold for statistical significance set at $P \leq 0.05$. All 
data were analysed using customized statistical spreadsheets (Microsoft Excel 2013,

244 Microsoft Corp, Redmond WA, USA).

\section{RESULTS}

Physiology

248 KTT had clear and trivial effects on oxygen cost and energy cost measures at $200 \mathrm{~W}$ that did not reach statistical significance. The effect of KTT on all other physiological parameters was unclear or unlikely, and not statistically significant (Table 1).

*** Insert Table $1 * * *$

\section{Kinematics}

255 The clear and likely effects of KTT on ROM values at $100 \mathrm{~W}$ (Table 2) and $200 \mathrm{~W}$ (Table

256 3) were trivial, except for the mean ankle ROM in the transverse plane at $100 \mathrm{~W}$ on the

257 dominant side, where a small non-significant increase was noted (ES, 0.35; $P$, 0.097; Table

258 2). In all other cases, the effect of KTT was unclear or unlikely, and not statistically

259 significant.

260

***Insert Table 2***

*** Insert Table $3 * * *$

\section{Electromyography}

The effect of KTT on certain VM, VM-to-VL ratio, BF, and RF-to-BF ratio measures were

267 clear, likely, and significant at $100 \mathrm{~W}$ (Table 4) and $200 \mathrm{~W}$ (Table 5). Changes primarily affected the efforts performed at $100 \mathrm{~W}$. 
270 At $100 \mathrm{~W}$, the effect of KTT on the non-dominant side was clear, likely, and significant for

271 increasing VM peak (ES, 1.35; $P, 0.044)$, and decreasing the RF-to-BF ratio peak (ES, -0.42;

$272 P, 0.021)$ and mean (ES, $-0.62 ; P, 0.016)$ measures. There was also clear and likely non-

273 significant increases in VM iEMG (ES, 0.72; $P$, 0.128); and VM-to-VL ratio peak (ES, 2.20;

$274 P, 0.118)$, iEMG (ES, $1.26 ; P, 0.097)$, and mean (ES, $1.21 ; P, 0.08)$ measures.

275

276 At $100 \mathrm{~W}$, the effect of KTT on the dominant side was clear, likely, and significant for

277 increasing VM iEMG (ES, 0.98; $P, 0.024)$ and mean (ES, 0.95; $P, 0.030)$; increasing VM-to-

278 VL ratio peak (ES, 2.19; $P, 0.009)$, iEMG (ES, 1.63; $P, 0.020)$, and mean (ES, 1.21; $P$,

279 0.029); and decreasing BF mean (ES, -0.36; $P, 0.047)$ measures. There was also a clear and

280 likely non-significant increase in VM peak (ES, 0.87; $P, 0.056)$; and decrease in RF peak

$281 \quad(\mathrm{ES},-0.39 ; P, 0.135)$ and mean $(\mathrm{ES},-0.51 ; P, 0.137)$ measures.

282

283

*** Insert Table 4***

At $200 \mathrm{~W}$, the effect of KTT on the non-dominant side was clear, likely, and significant for increasing VM iEMG (ES, 1.04; $P, 0.014)$. There was also a clear and likely non-significant increase in VM peak (ES, 0.92; $P, 0.122)$ and mean (ES, 0.92; $P, 0.088)$; increase in VM-toVL ratio peak (ES, 1.41; $P, 0.157)$, iEMG (ES, 0.88; $P, 0.124)$, and mean (ES, 2.07; $P$, 0.098); and decrease in BF mean (ES, $-0.39 ; P, 0.194)$. At $200 \mathrm{~W}$, there was a clear and likely non-significant effect of KTT on decreasing peak BF (ES, $-0.69 ; P, 0.077)$ measures.

***Insert Table 5***

\section{Perception}

295 Most cyclists perceived KTT as being comfortable, providing additional stability to the

296 knee, and enhancing performance (Figure 3). However, three cyclists felt that KTT was

297 uncomfortable, with one cyclist feeling more unstable with KTT. 


\section{DISCUSSION}

302 Despite most cyclists perceiving enhanced performance and knee stability with patella KTT; the effects of the intervention were either unclear, non-significant, or clearly trivial for all physiological and kinematic measures, except for a small non-significant increase in ankle

305 ROM on the dominant side in the transverse plane at $100 \mathrm{~W}$. KTT affected the EMG-

306 determined muscle activation patterns the most, notably increasing VM and VM-to-VL ratio

307 measures at both powers; and decreasing BF and RF-to-BF ratio measures. Overall, our

308 findings indicate a potential for patella KTT to alter the neuromuscular recruitment patterns

309 of elite cyclists with no current musculoskeletal injury at low powers, which could have

310 implications in the prevention of overuse injuries.

\section{$312 \quad$ Physiology}

313 Cycling biomechanics and neuromuscular function can alter energy cost, oxygen cost, and 314 cycling efficiency. For instance, cycling in a more aerodynamic than upright position can 315 increase oxygen cost by $1.5 \%$ (Gnehm et al., 1997). This increase is speculated to result in 316 part from a shift in mean hip-joint angles towards greater flexion, which alters the operating points of the hip- and knee-joint muscles on the force-velocity and force-length curves, as well as an increase in hip adductor activation to prevent out-of-plane motion in extreme hip

319 flexion. The biomechanical and neuromuscular differences associated with changing cycling 320 positions from aerodynamic to upright are inherently much larger than those potentially 321 resulting from KTT application, especially proximally at the trunk, pelvis, and hip (Dorel et 322 al., 2009). It is likely that the neuromuscular changes observed here in VM, VM-to-VL ratio, $323 \mathrm{BF}$, and RF-to-BF ratio measures with KTT were not sufficient to cause significant or clear alterations in the physiological parameters monitored. 


\section{$326 \quad$ Kinematics and muscle activation}

327 Ideally, the legs should act as pistons during cycling (Sanner and O'Halloran, 2000), with

328 lower-body motion mainly directed upwards and downwards, and cyclists in a saddle

329 position that allows knee extension with minimal valgus angulation. Most studies addressing

330 lower-body kinematics during cycling have focused on sagittal plane motion, with our

331 sagittal ROM values agreeing with those typically reported (Bini et al., 2011). Although a

332 certain amount of 'out-of-plane' motion is anticipated, lower-body misalignment and

333 excessive out-of-plane motion are reported to contribute to musculoskeletal injuries in

334 cyclists (Bini et al., 2011, Gregor and Wheeler, 1994). One of the proposed benefits of KTT

335 is to assist in joint alignment through improvements in proprioception, which in turn can

336 improve movement patterns and cycling efficiency. Hence, we anticipated less out-of-plane

337 motion at the knee with KTT; however, such a reduction was not evident.

339 Previous studies have shown that patellar taping can affect movement patterns in both

340 healthy and symptomatic individuals (Theobald et al., 2014), as well as muscle recruitment

341 of VM (Gilleard et al., 1998), VL (Gilleard et al., 1998), and RF (Konishi, 2013). These

342 changes in neuromuscular function are suggested to result from the tactile stimulation of the

343 skin (Konishi, 2013), rather than by the actual tape configuration or alterations in patellar

344 positioning (Bockrath et al., 1993). Conversely, several other studies have observed no

345 effect from therapeutic taping on neuromuscular function (Halski et al., 2015, Lins et al.,

346 2013), with little evidence supporting improved athletic performance or muscle strength

347 (Csapo and Alegre, 2015, Lins et al., 2013). Our results support the hypothesis that applying

348 KTT across the knee stimulates VM activation and increases the VM-to-VL ratio in

349 asymptomatic elite cyclists during submaximal efforts, without inducing significant or clear

350 changes in knee biomechanics.

352 The VM muscle is the dynamic medial stabilizer of the patella and functionally important in

353 aligning the patella within the patella-femoral joint trochlea, which cannot be readily 
examined using skin-markers and 3D motion capture. Our KTT application had no mechanical intent, and the altered muscle activation seen here most likely resulted from the enhanced tactile input that altered the excitability of the central nervous system and modulated proprioceptive afferent feedback loops (Simoneau et al., 1997). The enhanced VM activation seen in our cyclists when wearing KTT could be beneficial for preventing patellofemoral pain given that VM is important for the dynamic alignment of the patella. Studies have shown that individuals with PFP exhibit lower activity levels of all vastus muscles during walking (Powers et al., 1996) and VM-to-VL ratios across a range of functional and isometric contraction tasks (Souza and Gross, 1991). Furthermore, delayed onset of EMG activity of the VM in relation to VL $(-0.67 \mathrm{~ms})$ has been identified as a contributing factor to the development of PFP in one prospective study (Van Tiggelen et al., 2009). That said, prospective studies on this topic in elite cyclists are needed to confirm the prophylactic effect of patella KTT on knee injury occurrence in this population group.

Although the VM and VL muscles play a critical role in power output during cycling, there is also a high activation of the RF and BF muscles (Akima et al., 2005), with proper coactivation of the hamstrings, which has been suggested to reduce stress at the knee during cycling (So et al., 2005). Hence, reducing the RF-to-BF ratio may have meaningful clinical implications for athletes who exhibit imbalances between knee extensor and flexor strength, poor coordination, and non-optimal activation patterns (i.e., athletes who are quadriceps dominant). With KTT application; there was a clear, likely, and significant decrease in mean

375 BF signals on the dominant side, as well as and peak and mean RF-to-BF ratio values at 100

$376 \mathrm{~W}$ on the non-dominant side; with only a small non-significant decrease in RF-to-BF mean

377 observed at $200 \mathrm{~W}$. Despite our results indicating some potential for alterations in RF-to-BF

378 muscle activation patterns, larger sample sizes would be needed to confirm outcomes and implications of these changes. 
381 Most of the neuromuscular effects observed at $100 \mathrm{~W}$ became unclear and non-significant at

$382200 \mathrm{~W}$, pointing to an interaction effect between power output and neuromuscular responses

383 to taping. It is well established that muscle contraction forces increase primarily due to an

384 increase in the number of motor units active and associated firing rates in a non-linear

385 fashion (Merletti and Parker, 2004), with previous cycling studies showing progressive

386 increase in muscle activation with progressive loads from 150, 220, 290, and $370 \mathrm{~W}$

387 (Carpes et al., 2010a). It is plausible that the effect of KTT on the neuromuscular control

388 diminished with increased overall muscle recruitment, explaining the attenuated effects of

389 KTT at $200 \mathrm{~W}$; however, the underlying mechanisms are unclear given the paucity of

390 literature investigating the effect of KTT at different contraction levels and loads within a

391 given exercise.

393 The non-dominant and dominant holistically demonstrated comparable responses to KTT, although some clear effects and significant findings were only detected on one side. This

395 discrepancy might be linked to preferred movement patterns of our cyclists, previous injuries 396 with residual neuromuscular inhibition or muscle weakness, or our limited sample size that 397 reduced our statistical power. Most studies suggest that bilateral pedalling asymmetries in 398 terms of power, work, or force increase as the workload decreases (Carpes et al., 2010b), 399 which might explain some of the differential responses between legs that were observed.

400 However, given that work was controlled, and power and force not monitored, the 401 mechanistic reasons behind the between-leg differences remain undetermined.

\section{Perception}

404 Applying RockTape ${ }^{\mathrm{TM}}$ to the anterior aspects of the arms and legs and posterior aspects of 405 the neck and back has previously been shown to decrease 'overall' and 'chest' ratings of 406 perceived exertion of trained cyclists, but not alter 'arm' and 'leg' ratings of perceived exertions or gross efficiency (Miller et al., 2015). The physiological findings from this same

408 investigation were unable to support improved athletic performance with RockTape ${ }^{\mathrm{TM}}$ use 
409 (Miller et al., 2015). Although most of our cyclists perceived additional knee stability and

410 enhanced performance with KTT; the biomechanical and physiological findings were unable

411 to support that KTT improved knee stability or economy, with KTT application exerting

412 unclear, non-significant, or clearly trivial effects on knee ROM and physiological measures.

413 It is likely that our cyclists' perceptions result from the EMG changes observed or a placebo

414 effect (Mak et al., 2018). Nonetheless, KTT application may still provide some benefits to

415 certain cyclists given the changes observed in the EMG parameters, notably the increased

$416 \mathrm{VM}$ activation and alterations in the VM-to-VL and RF-to-BF activation ratios.

\section{$418 \quad$ Individual responses}

419 One cyclist perceived KTT as uncomfortable and decreasing knee stability. Nonetheless, this 420 particular cyclist felt that KTT improved his performance. This cyclist's data indicated a

421 slight worsening in cycling economy measures at $200 \mathrm{~W}$, with a general increase in knee 422 ROM in all planes of motion with KTT. Simultaneously, EMG signals for VM, VL, and RF, 423 and the VM-to-VL and RF-to-BF ratios increased with KTT, and decreased for BF. In this 424 particular case, perceptions matched well with biomechanical findings, but not necessarily 425 with the physiological ones. In contrast, several cyclists who perceived an increased knee 426 stability, an improved performance, and felt comfortable with KTT application showed 427 'negative' responses, with their perceptual ratings disagreeing with their objective measures. 428 Hence, although individual data suggest the presences of 'positive responders', 'negative 429 responders', and 'non-responders', we were unable to clearly define subgroups from the 430 subjective data collected.

Limitations

433 Small sample sizes are an inherent limitation in any high performance sport environment, 434 which reduced our statistical power. All male National Team cyclists available for testing 435 accepted to participate. Our sample size could not be increased further without 436 compromising the external validity of our findings (i.e., testing lower-level cyclists). Future 
research should examine the repeatability of the effect of KTT application and the potential

438 for any long-term effect or habituation to KTT more thoroughly. We tested only elite male

439 cyclists since the national-level female cyclists were training overseas at the time of data

440 collection and thus the findings may be specific to this population. Female athletes differ

441 physiologically, morphologically, and with respect to injury risk factors compared to male

442 athletes, therefore specific investigations of how female cyclists respond to KTT are

443 warranted. We also acknowledge that the power settings selected were submaximal for elite

444 cyclists and that responses at higher powers might differ. Using lower powers was a

445 necessity to calculate steady state oxygen consumption, economy, and efficiency, and for

446 practical relevance to the National Cycling Team of Malaysia. It should be noted however,

447 that during tour events cyclists often perform for prolonged periods at relatively low levels

448 of power production. For example, Alexander Kristoff's average power output during the

449 first hour of Stage 4 of the 2017 Tour de France was $118 \mathrm{~W}$ and his average power output

450 over the entire 4:53:54 of the stage (in which he finished second), was $189 \mathrm{~W}$

451 (www.trainingpeaks.com). Finally, given the minimalist taping technique applied, it was not

452 possible to implement a "placebo" taping method or different taping configurations to confer

453 differences in proprioceptive input.

\section{CONCLUSIONS}

456 Most cyclists perceived increased performance and knee stability with patella KTT, but the

457 intervention had little impact on physiological measures and mostly trivial non-significant

458 effects on knee ROM values. However, patella KTT decreased ROM at the pelvis and trunk

459 at the higher power and appeared to stabilize the segments proximally, which could be a

460 favourable adaptation in cyclists (McDaniel et al., 2005). KTT application did alter EMG

461 responses, notably increasing VM activation and altering the VM-to-VL activation ratio at

462100 and $200 \mathrm{~W}$, and changes indicating an increase in BF recruitment in relation to RF at

$463100 \mathrm{~W}$. Our findings imply that there is a potential for patella KTT to alter neuromuscular

464 recruitment patterns in elite uninjured cyclists, which could have implications for injury 
prevention and especially the development of PFP by assisting with patella alignment and

466 alleviating knee-joint stress through neuromuscular pathways as opposed to altering knee

467 biomechanics. As such, the neuromuscular changes we observed indicate that cyclists may

468 benefit acutely from patella KTT, although the longitudinal effects of KTT use have not yet

469 been established.

470

471 ACKNOWLEDGEMENTS

472 The authors would like to thank Dr. Yeo Wee Kian and Mohd Izham Bin Mohamad for

473 assistance in coordinating the project, Mee Chee Chong for helping during data collection,

474 and all cyclists for their voluntary participation.

475

476

477 REFERENCES

478 Akima H, Kinugasa R, Kuno S. Recruitment of the thigh muscles during sprint cycling by

479 muscle functional magnetic resonance imaging. Int J Sports Med. 2005;26:245-52.

480 Bell AL, Brand RA, Pedersen DR. Prediction of hip joint centre location from external

481 landmarks. Hum Mov Sci. 1989;8:3-16.

482 Bertucci W, Grappe F, Groslambert A. Laboratory versus outdoor cycling conditions:

483 Differences in pedaling biomechanics. J Appl Biomech. 2007;23:87-92.

484 Bertucci WM, Betik AC, Duc S, Grappe F. Gross efficiency and cycling economy are higher

485 in the field as compared with on an Axiom stationary ergometer. J Appl Biomech.

$486 \quad 2012 ; 28: 636-44$.

487 Bini R, Hume PA, Croft JL. Effects of bicycle saddle height on knee injury risk and cycling

488 performance. Sports Med. 2011;41:463-76.

489 Bockrath K, Wooden C, Worrell T, Ingersoll CD, Farr J. Effects of patella taping on patella

490 position and perceived pain. Med Sci Sports Exerc. 1993;25:989-92.

491 Cappozzo A, Cappello A, Croce UD, Pensalfini F. Surface-marker cluster design criteria for

492 3-d bone movement reconstruction. IEEE Trans Biomed Eng. 1997;44:1165-74. 
493 Carpes FP, Diefenthaeler F, Bini RR, Stefanyshyn DJ, Faria IE, Mota CB. Influence of leg

494 preference on bilateral muscle activation during cycling. J Sports Sci. 2010a;29:151-9.

495 Carpes FP, Mota CB, Faria IE. On the bilateral asymmetry during running and cycling - A

496 review considering leg preference. Phys Ther Sport. 2010b;11:136-42.

497 Coyle EF, Sidossis LS, Horowitz JF, Beltz JD. Cycling efficiency is related to the

498 percentage of type I muscle fibers. Med Sci Sports Exerc. 1992;24:782-8.

499 Csapo R, Alegre LM. Effects of Kinesio® taping on skeletal muscle strength—A meta-

500 analysis of current evidence. J Sci Med Sport. 2015;18:450-6.

501 Dorel S, Couturier A, Hug F. Influence of different racing positions on mechanical and

502 electromyographic patterns during pedalling. Scand J Med Sci Sports. 2009;19:44-54.

503 Gilleard W, McConnell J, Parsons D. The effect of patellar taping on the onset of vastus

504 medialis obliquus and vastus lateralis muscle activity in persons with patellofemoral pain.

505 Phys Ther. 1998;78:25-32.

506 Gnehm P, Reichenbach S, Altpeter E, Widmer H, Hoppeler H. Influence of different racing

507 positions on metabolic cost in elite cyclists. Med Sci Sports Exerc. 1997;29:818-23.

508 Gregor RJ, Wheeler JB. Biomechanical factors associated with shoe/pedal interfaces.

509 Implications for injury. Sports Med. 1994;17:117-31.

510 Grood ES, Suntay WJ. A joint coordinate system for the clinical description of three-

511 dimensional motions: Application to the knee. J Biomech Eng. 1983;105:136-44.

512 Halski T, Dymarek R, Ptaszkowski K, Słupska L, Rajfur K, Rajfur J, et al. Kinesiology

513 Taping does not modify electromyographic activity or muscle flexibility of quadriceps

514 femoris muscle: A randomized, placebo-controlled pilot study in healthy volleyball players.

515 Med Sci Monit. 2015;21:2232-9.

516 Hermens HJ, Freriks B, Disselhorst-Klug C, Rau G. Development of recommendations for

517 SEMG sensors and sensor placement procedures. J Electromyogr Kinesiol. 2000;10:361-74.

518 Jeukendrup AE, Wallis GA. Measurement of substrate oxidation during exercise by means

519 of gas exchange measurements. Int J Sports Med. 2005;26:S28-37. 
520 Konishi Y. Tactile stimulation with Kinesiology tape alleviates muscle weakness attributable 521 to attenuation of Ia afferents. J Sci Med Sport. 2013;16:45-8.

522 Likert R. A technique for the measurement of attitudes. Arch Psychol. 1932;22:5-55.

523 Lins CADA, Neto FL, de Amorim ABC, Macedo LdB, Brasileiro JS. Kinesio Taping® does

524 not alter neuromuscular performance of femoral quadriceps or lower limb function in

525 healthy subjects: Randomized, blind, controlled, clinical trial. Man Ther. 2013;18:41-5.

526 Lyman KJ, Keister K, Gange K, Mellinger CD, Hanson TA. Investigating the effectiveness

527 of Kinesio(R) taping space correction method in healthy adults on patellofemoral joint and

528 subcutaneous space. Int J Sports Phys Ther. 2017;12:250-7.

529 Mak DN, Au IP, Chan M, Chan ZY, An WW, Zhang JH, et al. Placebo effect of facilitatory

530 Kinesio tape on muscle activity and muscle strength. Physiother Theory Pract. 2018:1-6. doi:

$531 \quad 10.1080 / 09593985.2018 .1441936$

532 Matheus JPC, Zille RR, Gomide Matheus LB, Lemos TV, Carregaro RL, Shimano AC.

533 Comparison of the mechanical properties of therapeutic elastic tapes used in sports and

534 clinical practice. Phys Ther Sport. 2017;24:74-8.

535 McDaniel J, Subudhi A, Martin JC. Torso stabilization reduces the metabolic cost of

536 producing cycling power. Can J Appl Physiol. 2005;30:433-41.

537 Merino-Marban R, Mayorga-Vega D, Fernandez-Rodriguez E. Effect of kinesio tape

538 application on calf pain and ankle range of motion in duathletes. J Hum Kinet. 2013;37:129-

53935.

540 Merletti R, di Torino P. Standards for reporting EMG data. J Electromyogr Kinesiol.

541 1999;9:III-IV.

542 Merletti R, Parker P. Electromyography: Physiology, Engineering, and Noninvasive

543 Applications. Hoboken, NJ: IEEE/John Wiley \& Sons; 2004.

544 Miller MG, Michael TJ, Nicholson KS, Petro RV, Hanson NJ, Prater DR. The effect of

545 Rocktape on rating of perceived exertion and cycling efficiency. J Strength Cond Res.

$546 \quad 2015 ; 29: 2608-12$. 
Ouyang JH, Chang KH, Hsu WY, Cho YT, Liou TH, Lin YN. Non-elastic taping, but not

548 elastic taping, provides benefits for patients with knee osteoarthritis: Systemic review and 549 meta-analysis. Clin Rehabil. 2018;32:3-17.

550 Powers CM, Landel R, Perry J. Timing and Intensity of Vastus Muscle Activity During

551 Functional Activities in Subjects With and Without Patellofemoral Pain. Phys Ther.

$552 \quad 1996 ; 76: 946-55$.

553 Reneker JC, Latham L, McGlawn R, Reneker MR. Effectiveness of kinesiology tape on

554 sports performance abilities in athletes: A systematic review. Phys Ther Sport. 2018;31:83-

55598.

556 Sanner W, O'Halloran W. The biomechanics, etiology, and treatment of cycling injuries. J

557 Am Podiatr Med Assoc. 2000;90:354-76.

558 Silberman MR. Bicycling injuries. Curr Sports Med Rep. 2013;12:337-45.

559 Simoneau GG, Degner RM, Kramper CA, Kittleson KH. Changes in ankle joint

560 proprioception resulting from strips of athletic tape applied over the skin. J Athl Train.

$561 \quad 1997 ; 32: 141$.

562 Smith TB, Hopkins WG. Variability and predictability of finals times of elite rowers. Med

563 Sci Sports Exerc. 2011;43:2155-60.

564 So RCH, Ng JKF, Ng GYF. Muscle recruitment pattern in cycling: A review. Phys Ther 565 Sport. 2005;6:89-96.

566 Souza DR, Gross MT. Comparison of vastus medialis obliquus: vastus lateralis muscle

567 integrated electromyographic ratios between healthy subjects and patients with

568 patellofemoral pain. Phys Ther. 1991;71:310-6.

569 Theobald G, Parry S, Richards J, Thewlis D, Tootill I, Selfe J. Biomechanical effects of

570 different treatment modalities used in knee pain during cycling. Physiother Pract Res.

$571 \quad 2012 ; 33: 16-21$.

572 Theobald G, Selfe J, Richards J, Roddam H. An investigation of the biomechanical efficacy

573 and clinical effectiveness of patello-femoral taping in elite and experienced cyclists. J Sci

574 Cycling. 2014;3:57-63. 
575 Van Tiggelen D, Cowan S, Coorevits P, Duvigneaud N, Witvrouw E. Delayed vastus

576 medialis obliquus to vastus lateralis onset timing contributes to the development of

577 patellofemoral pain in previously healthy men: A prospective study. Am J Sports Med.

$578 \quad 2009 ; 37: 1099-105$.

579 Williams S, Whatman C, Hume PA, Sheerin K. Kinesio taping in treatment and prevention

580 of sports injuries. Sports Med. 2012;42:153-64.

581 Zech A, Wellmann K. Perceptions of football players regarding injury risk factors and

582 prevention strategies. PLoS One. 2017;12:e0176829. 
583 Table 1. Mean \pm SD values for oxygen cost $(\mathrm{mL} / \mathrm{kg} / \mathrm{km})$, energy cost $(\mathrm{kcal} / \mathrm{kg} / \mathrm{km})$, cycling 584 economy (W/L/min), heart rate (bpm), and gross efficiency (\%) in the No Tape (NT) and

585 Kinesiology-Type Tape (KTT) conditions during the 100 and $200 \mathrm{~W}$ cycling efforts. Delta

586 efficiency (\%) in NT and KTT conditions is also presented. Differences between conditions

587 are expressed using mean change $\left(\Delta_{\text {mean }}\right)$; standardized effect size (ES); and paired $t$-test

588 statistical significance values $(P)$. Thresholds for clear ES are provided (trivial, small, large,

589 and very large) and significant changes $(P \leq 0.05)$ are highlighted in grey.

\begin{tabular}{|c|c|c|c|c|c|}
\hline Par & NT & KTT & $\Delta_{\text {mean }}$ & ES (threshold) & $P$ \\
\hline & \multicolumn{5}{|c|}{$100 \mathrm{~W}$} \\
\hline Oxygen cost & $175.4 \pm 30.6$ & $175.2 \pm 33.5$ & $-0.2 \pm 14.1$ & $-0.01 \pm 0.42$ (unclear) & 0.963 \\
\hline Energy cost & \pm 0.15 & $0.86 \pm 0.16$ & $0.001 \pm 0.07$ & $0.001 \pm 0.43$ (unclear) & 0.993 \\
\hline Economy & $53.4 \pm 5.6$ & $53.6 \pm 5.5$ & $0.2 \pm 4.5$ & $0.03 \pm 0.78$ (unclear) & 0.885 \\
\hline Heart rate & $115.3 \pm 9.8$ & $115.6 \pm 9.5$ & $0.3 \pm 5.8$ & $0.03 \pm 0.58$ (unclear) & 0.845 \\
\hline \multirow[t]{2}{*}{ Gross efficiency } & $5.5 \pm 1.6$ & $15.6 \pm 1.6$ & $0.04 \pm 1.31$ & $0.02 \pm 0.79$ (unclear) & 0.926 \\
\hline & \multicolumn{5}{|c|}{$200 \mathrm{~W}$} \\
\hline Oxygen cost & $277.3 \pm 48.0$ & $273.4 \pm 46.1$ & $-3.9 \pm 12.6$ & $-0.08 \pm 0.26{\text { (trivial })^{\S}}^{\S}$ & 0.311 \\
\hline Energy cost & $1.37 \pm 0.23$ & $1.35 \pm 0.22$ & $-0.02 \pm 0.06$ & $-0.09 \pm 0.26{\text { (trivial })^{\S}}^{\S}$ & 0.263 \\
\hline Economy & $67.4 \pm 5.2$ & $68.4 \pm 5.9$ & $1.0 \pm 3.1$ & $0.19 \pm 0.54($ trivial $)$ & 0.303 \\
\hline Heart rate & $147.6 \pm 9.2$ & $146.8 \pm 8.4$ & $-0.8 \pm 6.4$ & $-0.08 \pm 0.70$ (unclear) & 0.691 \\
\hline Gross efficiency & $19.5 \pm 1.5$ & $19.8 \pm 1.7$ & $0.3 \pm 0.9$ & $0.21 \pm 0.54($ small $)$ & 0.260 \\
\hline Delta efficiency & $26.5 \pm 3.1$ & $27.3 \pm 2.3$ & $0.8 \pm 3.0$ & $0.25 \pm 1.04$ (unclear) & 0.380 \\
\hline
\end{tabular}

590 Note. An effect was deemed 'unclear' when its $90 \%$ confidence limit overlapped the

591 thresholds for small positive and small negative effects (i.e., 5\%). ${ }^{\$}$ Probability of the effect

592 exceeds $75 \%$ and is 'likely' to be clinically meaningful. 
593 Table 2. Mean \pm SD values for range of motion values $\left(^{\circ}\right)$ in the sagittal $(\mathrm{X})$, coronal $(\mathrm{Y})$, and transverse (Z) planes in the No Tape (NT) and Kinesiology-Type Tape (KTT) conditions during the $100 \mathrm{~W}$ cycling efforts for the dominant (D) and non-dominant (ND) sides. Differences between conditions are expressed using mean change $\left(\Delta_{\text {mean }}\right)$; standardized effect size (ES); and paired $t$-test statistical significance values $(P)$. Thresholds for clear ES are provided (trivial, small, large, and very large) and significant changes $(P \leq 0.05)$ are highlighted in grey.

\begin{tabular}{|c|c|c|c|c|c|c|c|}
\hline Joint & Side & Plane & NT & KTT & $\Delta_{\text {mean }}$ & ES & $P$ \\
\hline \multirow{6}{*}{ Ankle } & \multirow{3}{*}{ ND } & $\mathbf{X}$ & $19.5 \pm 8.1$ & $19.9 \pm 8.7$ & $0.4 \pm 2.1$ & 0.05 (trivial) $^{\S}$ & 0.517 \\
\hline & & $\mathbf{Y}$ & $5.3 \pm 1.7$ & $5.5 \pm 1.9$ & $0.2 \pm 0.6$ & 0.11 (trivial) $^{\S}$ & 0.289 \\
\hline & & $\mathbf{Z}$ & $5.7 \pm 2.3$ & $5.7 \pm 1.9$ & $0.0 \pm 1.5$ & 0.02 (unclear) & 0.920 \\
\hline & \multirow{3}{*}{ D } & $\mathbf{X}$ & $21.4 \pm 4.9$ & $20.3 \pm 6.0$ & $-1.1 \pm 3.9$ & -0.22 (small) & 0.355 \\
\hline & & $\mathbf{Y}$ & $4.3 \pm 1.8$ & $5.0 \pm 1.7$ & $0.6 \pm 1.2$ & $0.35{\text { (small })^{\S}}^{\S}$ & 0.097 \\
\hline & & $\mathbf{Z}$ & $5.5 \pm 0.7$ & $5.2 \pm 1.0$ & $-0.3 \pm 1.1$ & -0.44 (unclear) & 0.350 \\
\hline \multirow{6}{*}{ Knee } & \multirow{3}{*}{ ND } & $\mathbf{X}$ & $76.7 \pm 3.1$ & $76.2 \pm 3.6$ & $-0.5 \pm 1.5$ & -0.16 (trivial) & 0.270 \\
\hline & & $\mathbf{Y}$ & $9.2 \pm 3.6$ & $9.0 \pm 3.3$ & $-0.2 \pm 1.5$ & $-0.04{\text { (trivial })^{\S}}^{\S}$ & 0.727 \\
\hline & & $\mathbf{Z}$ & $9.2 \pm 4.9$ & $9.3 \pm 3.9$ & $0.1 \pm 2.6$ & 0.03 (unclear) & 0.867 \\
\hline & \multirow{3}{*}{ D } & $\mathbf{X}$ & $78.8 \pm 2.3$ & $78.2 \pm 2.2$ & $-0.5 \pm 1.7$ & -0.23 (small) & 0.304 \\
\hline & & $\mathbf{Y}$ & $8.3 \pm 2.6$ & $8.1 \pm 2.3$ & $-0.2 \pm 1.1$ & $-0.08{\text { (trivial })^{\S}}^{\S}$ & 0.526 \\
\hline & & $\mathbf{Z}$ & $12.3 \pm 5.3$ & $12.4 \pm 4.7$ & $0.0 \pm 1.8$ & 0.003 (trivial) $^{\S}$ & 0.976 \\
\hline \multirow{6}{*}{ Hip } & \multirow{3}{*}{ ND } & $\mathbf{X}$ & $48.7 \pm 3.5$ & $48.8 \pm 3.8$ & $0.1 \pm 1.5$ & 0.02 (unclear) & 0.901 \\
\hline & & $\mathbf{Y}$ & $6.3 \pm 3.4$ & $6.2 \pm 2.8$ & $0.0 \pm 1.3$ & -0.01 (trivial) $^{\S}$ & 0.915 \\
\hline & & $\mathbf{Z}$ & $11.5 \pm 3.8$ & $11.4 \pm 4.3$ & $0.0 \pm 1.8$ & -0.01 (unclear) & 0.937 \\
\hline & \multirow{3}{*}{ D } & $\mathbf{X}$ & $47.5 \pm 2.9$ & $47.4 \pm 2.8$ & $-0.1 \pm 1.1$ & -0.04 (trivial) $^{\S}$ & 0.694 \\
\hline & & $\mathbf{Y}$ & $6.6 \pm 1.8$ & $6.6 \pm 1.9$ & $0.0 \pm 1.4$ & -0.005 (unclear) & 0.983 \\
\hline & & $\mathbf{Z}$ & $10.0 \pm 3.7$ & $9.5 \pm 3.2$ & $-0.5 \pm 1.4$ & -0.12 (trivial) & 0.283 \\
\hline \multirow{3}{*}{ Pelvis } & & $\mathbf{X}$ & $8.1 \pm 1.8$ & $8.3 \pm 2.2$ & $0.2 \pm 1.4$ & 0.10 (unclear) & 0.647 \\
\hline & & $\mathbf{Y}$ & $3.5 \pm 0.9$ & $3.6 \pm 1.1$ & $0.1 \pm 0.5$ & 0.09 (trivial) & 0.561 \\
\hline & & $\mathbf{Z}$ & $3.7 \pm 1.9$ & $3.6 \pm 1.3$ & $-0.1 \pm 2.2$ & -0.05 (unclear) & 0.881 \\
\hline \multirow{3}{*}{ Trunk } & & $\mathbf{X}$ & $8.8 \pm 3.9$ & $10.1 \pm 8.2$ & $1.3 \pm 8.9$ & 0.34 (unclear) & 0.620 \\
\hline & & $\mathbf{Y}$ & $0.8 \pm 0.2$ & $0.7 \pm 0.2$ & $0.0 \pm 0.1$ & $-0.07{\text { (trivial })^{\S}}^{\S}$ & 0.643 \\
\hline & & $\mathbf{Z}$ & $8.4 \pm 4.1$ & $9.6 \pm 8.5$ & $1.2 \pm 9.4$ & 0.30 (unclear) & 0.663 \\
\hline
\end{tabular}

Notes. Sagittal (X): ankle dorsiflexion and plantar flexion, knee and hip flexion and

601 extension, pelvis and trunk anterior and posterior tilt; Coronal (Y): ankle inversion and eversion, knee valgus and varus, hip abduction and adduction, pelvis and trunk nondominant side and dominant side tilt; Transverse (Z) ankle, knee, and hip internal and external rotation, pelvis and trunk non-dominant side and dominant side rotation. An effect was deemed 'unclear' when its $90 \%$ confidence limit overlapped the thresholds for small 606 positive and small negative effects (i.e., 5\%). ${ }^{\S}$ Probability of the effect exceeds $75 \%$ and is 607 'likely' to be clinically meaningful. 
608 Table 3. Mean \pm SD values for range of motion values $\left(^{\circ}\right)$ in the sagittal $(\mathrm{X})$, coronal $(\mathrm{Y})$,

609 and transverse (Z) planes in the No Tape (NT) and Kinesiology-Type Tape (KTT)

610 conditions during the $200 \mathrm{~W}$ cycling efforts for the dominant (D) and non-dominant (ND)

611 sides. Differences between conditions are expressed using mean change ( $\left.\Delta_{\text {mean }}\right)$; standardized

612 effect size (ES); and paired $t$-test statistical significance values $(P)$. Thresholds for clear ES

613 are provided (trivial, small, large, and very large) and significant changes $(P \leq 0.05)$ are

614 highlighted in grey.

\begin{tabular}{|c|c|c|c|c|c|c|c|}
\hline Joint & Side & Plane & NT & $\overline{\text { KTT }}$ & $\overline{\Delta_{\text {mean }}}$ & ES & $\bar{P}$ \\
\hline \multirow{6}{*}{ Ankle } & \multirow{3}{*}{ ND } & $\bar{X}$ & $21.7 \pm 7.8$ & $22.7 \pm 8.3$ & $1.0 \pm 2.6$ & 0.12 (trivial) $^{\S}$ & 0.235 \\
\hline & & $\mathbf{Y}$ & $5.9 \pm 2.0$ & $6.2 \pm 2.6$ & $0.2 \pm 1.0$ & 0.11 (trivial) & 0.450 \\
\hline & & $\mathbf{Z}$ & $5.9 \pm 1.8$ & $5.7 \pm 1.9$ & $-0.2 \pm 1.0$ & -0.11 (trivial) & 0.529 \\
\hline & \multirow{3}{*}{ D } & $\mathbf{X}$ & $24.0 \pm 6.9$ & $24.7 \pm 5.7$ & $0.7 \pm 3.0$ & 0.10 (trivial) $^{\S}$ & 0.435 \\
\hline & & $\mathbf{Y}$ & $4.7 \pm 1.3$ & $4.9 \pm 1.5$ & $0.2 \pm 0.6$ & 0.14 (trivial) & 0.342 \\
\hline & & $\mathbf{Z}$ & $6.0 \pm 1.6$ & $5.8 \pm 1.7$ & $-0.2 \pm 0.4$ & -0.14 (trivial) $^{\S}$ & 0.123 \\
\hline \multirow{6}{*}{ Knee } & \multirow{3}{*}{ ND } & $\mathbf{X}$ & $78.4 \pm 3.7$ & $78.9 \pm 3.1$ & $0.5 \pm 1.7$ & 0.14 (trivial) & 0.302 \\
\hline & & $\mathbf{Y}$ & $8.3 \pm 2.6$ & $8.2 \pm 2.6$ & $-0.7 \pm 1.4$ & -0.03 (unclear) & 0.849 \\
\hline & & $\mathbf{Z}$ & $9.4 \pm 4.1$ & $8.8 \pm 3.1$ & $-0.6 \pm 1.8$ & -0.14 (trivial) & 0.277 \\
\hline & \multirow{3}{*}{ D } & $\mathbf{X}$ & $80.1 \pm 2.3$ & $80.9 \pm 2.5$ & $0.8 \pm 2.1$ & 0.34 (small) & 0.224 \\
\hline & & $\mathbf{Y}$ & $7.7 \pm 2.1$ & $8.1 \pm 2.1$ & $0.4 \pm 0.9$ & 0.19 (trivial) & 0.146 \\
\hline & & $\mathbf{Z}$ & $11.5 \pm 4.5$ & $11.2 \pm 4.5$ & $-0.3 \pm 1.6$ & -0.07 (trivial) $^{\S}$ & 0.496 \\
\hline \multirow{6}{*}{ Hip } & \multirow{3}{*}{ ND } & $\mathbf{X}$ & $49.6 \pm 3.9$ & $49.3 \pm 4.5$ & $-0.9 \pm 2.1$ & -0.07 (unclear) & 0.661 \\
\hline & & $\mathbf{Y}$ & $7.4 \pm 3.7$ & $7.4 \pm 3.4$ & $0.0 \pm 1.0$ & $0.001{\text { (trivial })^{\S}}^{\S}$ & 0.988 \\
\hline & & $\mathbf{Z}$ & $11.5 \pm 3.4$ & $12.1 \pm 3.4$ & $0.6 \pm 1.7$ & 0.18 (trivial) & 0.245 \\
\hline & \multirow{3}{*}{ D } & $\mathbf{X}$ & $47.4 \pm 2.8$ & $47.5 \pm 2.4$ & $0.1 \pm 1.4$ & 0.02 (unclear) & 0.874 \\
\hline & & $\mathbf{Y}$ & $7.1 \pm 2.5$ & $6.9 \pm 2.2$ & $-0.3 \pm 1.2$ & -0.10 (trivial) & 0.490 \\
\hline & & $\mathbf{Z}$ & $10.3 \pm 3.1$ & $10.4 \pm 3.2$ & $0.1 \pm 1.5$ & 0.03 (unclear) & 0.812 \\
\hline \multirow{3}{*}{ Pelvis } & & $\bar{X}$ & $8.4 \pm 2.4$ & $7.9 \pm 2.2$ & $-0.5 \pm 1.1$ & -0.19 (trivial) & 0.178 \\
\hline & & $\mathbf{Y}$ & $3.5 \pm 1.3$ & $3.7 \pm 1.5$ & $0.2 \pm 0.5$ & 0.14 (trivial) & 0.269 \\
\hline & & $\mathbf{Z}$ & $4.0 \pm 1.8$ & $3.6 \pm 1.1$ & $-0.4 \pm 1.8$ & -0.22 (unclear) & 0.447 \\
\hline \multirow{3}{*}{ Trunk } & & $\bar{X}$ & $9.5 \pm 4.2$ & $8.6 \pm 2.7$ & $-0.9 \pm 3.4$ & -0.21 (unclear) & 0.391 \\
\hline & & $\mathbf{Y}$ & $0.9 \pm 0.5$ & $0.9 \pm 0.3$ & $0.0 \pm 0.3$ & -0.04 (unclear) & 0.834 \\
\hline & & $\mathbf{Z}$ & $8.6 \pm 3.8$ & $7.8 \pm 2.6$ & $-0.9 \pm 3.6$ & -0.22 (unclear) & 0.430 \\
\hline
\end{tabular}

615 Notes. Sagittal (X): ankle dorsiflexion and plantar flexion, knee and hip flexion and

616 extension, pelvis and trunk anterior and posterior tilt; Coronal (Y): ankle inversion and

617 eversion, knee valgus and varus, hip abduction and adduction, pelvis and trunk non-

618 dominant side and dominant side tilt; Transverse (Z) ankle, knee, and hip internal and

619 external rotation, pelvis and trunk non-dominant side and dominant side rotation. An effect

620 was deemed 'unclear' when its $90 \%$ confidence limit overlapped the thresholds for small

621 positive and small negative effects (i.e., 5\%). ${ }^{\S}$ Probability of the effect exceeds $75 \%$ and is

622 'likely' to be clinically meaningful. 
624 Table 4. Mean $\left(\%_{\max }\right)$, peak $\left(\%_{\max }\right)$, and integrated EMG (iEMG, $\left.\%_{\max }\right)$ signal values (mean

$625 \pm \mathrm{SD}$ ) for the vastus medialis (VM), vastus lateralis (VL), rectus femoris (RF), and biceps

626 femoris (BF) muscles in the No Tape (NT) and Kinesiology-Type Tape (KTT) conditions

627 during the $100 \mathrm{~W}$ cycling efforts for the dominant (D) and non-dominant (ND) sides. The

628 VM-to-VL and RF-to-BF activation ratios are also presented. Differences between

629 conditions are expressed using mean change $\left(\Delta_{\text {mean }}\right)$; standardized effect size (ES); and

630 paired $t$-test statistical significance values $(P)$. Thresholds for clear ES are provided (trivial,

631 small, large, and very large) and significant changes $(P \leq 0.05)$ are highlighted in grey.

\begin{tabular}{|c|c|c|c|c|c|c|c|}
\hline Muscle & Side & EMG & NT & KTT & $\Delta_{\text {mean }}$ & ES (threshold) & $P$ \\
\hline \multirow{6}{*}{ VM } & \multirow{3}{*}{ ND } & Peak & $29.5 \pm 5.9$ & $37.4 \pm 5.1$ & $8.0 \pm 7.4$ & $1.35{\text { (large })^{\S}}^{\S}$ & 0.047 \\
\hline & & iEMG & $39.9 \pm 10.1$ & $47.3 \pm 7.9$ & $7.3 \pm 9.9$ & $0.72(\mathrm{~m}$ & 0.128 \\
\hline & & Mean & $9.4 \pm 1.7$ & $9.2 \pm 2.6$ & $-0.2 \pm 2.6$ & -0.13 (unclear) & 0.840 \\
\hline & \multirow{3}{*}{ D } & Peak & $31.4 \pm 9.3$ & 39.5 & $.1 \pm 10.1$ & 0.87 & 0.056 \\
\hline & & iEMG & $38.1 \pm$ & $49.7 \pm$ & $11.7 \pm 8.9$ & 0.98 & 0.024 \\
\hline & & Mean & $7.9 \pm 2.1$ & $9.9 \pm 2.2$ & $2.0 \pm 2.1$ & $0.95{\text { (moderate })^{\S}}^{\S}$ & 0.030 \\
\hline \multirow{6}{*}{ VL } & \multirow{3}{*}{ ND } & Peak & $39.3 \pm$ & 36.8 & $-2.5 \pm 15.8$ & -0.2 & 0.717 \\
\hline & & iEMG & $47.3 \pm$ & 36.8 & $-0.5 \pm 18.0$ & -0.0 & 0.950 \\
\hline & & Mean & $9.4 \pm 1.7$ & $9.2=$ & $-0.2 \pm 2.6$ & -0 . & 0.840 \\
\hline & \multirow{3}{*}{ D } & Peak & $37.0=$ & 36.9 & $-0.2 \pm 10.0$ & & 0.965 \\
\hline & & iEMG & $44.7 \pm$ & $47.2=$ & $2.5 \pm 10.2$ & & 0.545 \\
\hline & & Mean & $8.7 \pm$ & 9.0 & $0.2 \pm 1.8$ & & 0.725 \\
\hline \multirow{6}{*}{$\mathbf{R F}$} & \multirow{3}{*}{ ND } & Peak & $24.7=$ & 22.7 & $-2.0 \pm 5.7$ & & 0.482 \\
\hline & & iEMG & 35.1 & 33.0 & $-2.1 \pm 4.8$ & & 0.391 \\
\hline & & Mean & $7.5 \pm 1.0$ & $7.0=$ & $-0.6 \pm 1.0$ & & 0.279 \\
\hline & \multirow{3}{*}{ D } & Peak & $29.6 \pm 11.0$ & 25.2 & $-4.3 \pm 4.3$ & & 0.135 \\
\hline & & iEMG & $39.0 \pm$ & 36.1 & $-3.0 \pm 4.6$ & -0.3 & 0.287 \\
\hline & & Mean & $7.3 \pm 1.3$ & $6.7 \pm 0.7$ & $-0.7 \pm 0.6$ & -0 . & 0.137 \\
\hline \multirow{6}{*}{ BF } & \multirow{3}{*}{ ND } & Peak & $2 \pm$ & 39.2 & $1.9 \pm 6.0$ & & 0.514 \\
\hline & & iEMG & $42.8 \pm$ & 42.8 & $-0.9 \pm 9.3$ & -0. & 0.830 \\
\hline & & Mean & $7.9 \pm$ & $8.4 \pm$ & $0.5 \pm 1.1$ & 0.2 & 0.351 \\
\hline & \multirow{3}{*}{ D } & Peak & $42.0=$ & 8.5 & $-3.5 \pm 3.2$ & -0.67 & 0.120 \\
\hline & & iEMG & $42.7=$ & 40.7 & $-2.1 \pm 3.3$ & -0.4 & 0.303 \\
\hline & & Mean & $7.6 \pm$ & $7.4=$ & $-0.6 \pm 0.3$ & -0 . & 0.044 \\
\hline \multirow{6}{*}{ VM:VL } & \multirow{3}{*}{ ND } & Peak & $81.6=$ & 12.2 & $17.6 \pm 19.8$ & 2.20 & 0.118 \\
\hline & & iEMG & 6.0 & 18.3 & $20.1 \pm 24.2$ & 1. & 0.097 \\
\hline & & Mean & $76.8 \pm 11.0$ & $95.4 \pm 14.2$ & $18.6 \pm 22.8$ & 0.95 & 0.088 \\
\hline & \multirow{3}{*}{ D } & Peak & $75.1 \pm 14.7$ & $107.2 \pm 29.2$ & $32.1 \pm 18.8$ & $2.19(1$ & 0.009 \\
\hline & & iEMG & $84.9 \pm 14.0$ & $107.7 \pm 26.0$ & $22.8 \pm 16.6$ & $1.63{\text { (large })^{\S}}^{\S}$ & 0.020 \\
\hline & & Mean & $91.9 \pm 19.6$ & $115.6 \pm 27.7$ & $23.7 \pm 23.4$ & $1.21{\text { (large })^{\S}}^{8}$ & 0.029 \\
\hline \multirow{6}{*}{ RF:BF } & \multirow{3}{*}{ ND } & Peak & $68.0 \pm 21.8$ & $58.8 \pm 18.7$ & $-9.1 \pm 6.5$ & $-0.42(\text { small })^{\S}$ & 0.021 \\
\hline & & iEMG & $85.6 \pm 19.8$ & $80.7 \pm 16.0$ & $-5.0 \pm 13.1$ & -0.2 & 0.445 \\
\hline & & Mean & $99.5 \pm 22.8$ & $85.5 \pm 17.2$ & $-14.0 \pm 7.8$ & $-0.62(\text { moderate })^{\S}$ & 0.016 \\
\hline & \multirow{3}{*}{ D } & & $69.5 \pm 18.8$ & $65.4 \pm$ & $-4.1 \pm 8.1$ & -0.2 & 0.388 \\
\hline & & iEMG & 91 & 89.5 & $-2.1 \pm 5.7$ & & 0.514 \\
\hline & & Mean & $93.9 \pm 23.6$ & $91.6 \pm 18.2$ & $-2.3 \pm 5.5$ & -0.10 (trivial) & 0.456 \\
\hline
\end{tabular}

Note. An effect was deemed 'unclear' when its $90 \%$ confidence limit overlapped the

633 thresholds for small positive and small negative effects (i.e., 5\%). An effect was deemed

634 'unclear' when its $90 \%$ confidence limit overlapped the thresholds for small positive and 


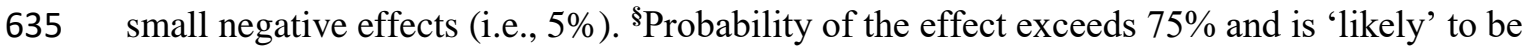
636 clinically meaningful. 
637 Table 5. Mean $\left(\%_{\max }\right)$, peak $\left(\%_{\max }\right)$, and integrated EMG (iEMG, $\left.\%_{\max }\right)$ signal values (mean $638 \pm \mathrm{SD}$ ) for the vastus medialis (VM), vastus lateralis (VL), rectus femoris (RF), and biceps

639 femoris (BF) muscles in the No Tape (NT) and Kinesiology-Type Tape (KTT) conditions

640 during the $200 \mathrm{~W}$ cycling efforts for the dominant (D) and non-dominant (ND) sides. The

641 VM-to-VL and RF-to-BF activation ratios are also presented. Differences between

642 conditions are expressed using mean change $\left(\Delta_{\text {mean }}\right)$; standardized effect size (ES); and

643 paired $t$-test statistical significance values $(P)$. Thresholds for clear ES are provided (trivial,

644 small, large, and very large) and significant changes $(P \leq 0.05)$ are highlighted in grey.

\begin{tabular}{|c|c|c|c|c|c|c|c|}
\hline Muscle & Side & EMG & NT & KTT & $\Delta_{\text {mean }}$ & ES (threshold) & $P$ \\
\hline \multirow{6}{*}{ VM } & \multirow{3}{*}{ ND } & Peak & $39.4 \pm 9.4$ & $48.0 \pm 4.4$ & $8.6 \pm 12.7$ & 0.92 (moderate) $^{\S}$ & 0.122 \\
\hline & & iEMG & $58.2 \pm 9.6$ & $68.1 \pm 5.7$ & $9.9 \pm 7.7$ & 1.04 (moderate) $)^{\S}$ & 0.014 \\
\hline & & Mean & $10.7 \pm 1.5$ & $12.1 \pm 1.8$ & $1.4 \pm 1.8$ & $0.92(\text { moderate })^{\S}$ & 0.088 \\
\hline & \multirow{3}{*}{ D } & Peak & $47.0 \pm 9.4$ & $48.2 \pm 5.3$ & $1.2 \pm 10.9$ & 0.13 (unclear) & 0.741 \\
\hline & & iEMG & $69.1 \pm 10.7$ & $70.1 \pm 6.2$ & $1.0 \pm 11.4$ & 0.09 (unclear) & 0.830 \\
\hline & & Mean & $13.0 \pm 2.4$ & $14.1 \pm 1.7$ & $1.1 \pm 3.0$ & 0.46 (unclear) & 0.380 \\
\hline \multirow{6}{*}{ VL } & \multirow{3}{*}{ ND } & Peak & $46.4 \pm 9.5$ & $47.7 \pm 6.2$ & $1.3 \pm 12.4$ & 0.13 (unclear) & 0.797 \\
\hline & & iEMG & $66.4 \pm 6.9$ & $68.9 \pm 11.5$ & $2.5 \pm 13.7$ & 0.37 & 0.643 \\
\hline & & Mean & $12.8 \pm 1.8$ & $12.8 \pm 3.2$ & $0.1 \pm 2.2$ & 0.05 (unclear) & 0.924 \\
\hline & \multirow{3}{*}{ D } & Peak & $50.2 \pm 7.0$ & $47.7 \pm 9.0$ & $-2.6 \pm 9.3$ & -0.37 & 0.433 \\
\hline & & iEMG & $65.8 \pm 8.1$ & $69.4 \pm 9.0$ & $3.6 \pm 9.4$ & 0.45 (unclear) & 0.284 \\
\hline & & Mean & $13.0 \pm 1.6$ & $13.1 \pm 2.1$ & $0.1 \pm 1.7$ & 0.09 & 0.831 \\
\hline \multirow{6}{*}{ RF } & \multirow{3}{*}{ ND } & Peak & $34.4 \pm 12.6$ & $37.1 \pm 2.5$ & $2.8 \pm 14.5$ & & 0.657 \\
\hline & & iEMG & $54.4 \pm 15.9$ & $53.7 \pm 6.7$ & $-0.7 \pm 15.1$ & ar) & 0.906 \\
\hline & & Mean & $11.9 \pm 3.0$ & $11.9 \pm 1.7$ & $-0.1 \pm 3.4$ & ear) & 0.971 \\
\hline & \multirow{3}{*}{ D } & Peak & $39.5 \pm 10.1$ & $40.8 \pm 6.6$ & $1.3 \pm 7.2$ & ear) & 0.672 \\
\hline & & iEMG & $62.1 \pm 11.9$ & $63.7 \pm 7.8$ & $1.6 \pm 13.6$ & 0.14 (unc & 0.761 \\
\hline & & Mean & $11.6 \pm 1.7$ & $11.4 \pm 1.8$ & $-0.2 \pm 2.2$ & -0.11 (unclear) & 0.844 \\
\hline \multirow{6}{*}{ BF } & \multirow{3}{*}{ ND } & Peak & $42.6 \pm 11.6$ & $45.1 \pm 6.4$ & $2.6 \pm 16.0$ & & 0.711 \\
\hline & & iEMG & $57.8 \pm 12.9$ & $58.8 \pm 8.7$ & $1.0 \pm 12.9$ & 0.0 & 0.846 \\
\hline & & Mean & $12.7 \pm 4.8$ & $12.2 \pm 3.0$ & $-0.5 \pm 3.7$ & -0.11 (unclear) & 0.735 \\
\hline & \multirow{3}{*}{ D } & Peak & $44.1 \pm 8.1$ & $38.5 \pm 5.9$ & $-5.6 \pm 6.2$ & $-0.69(\text { moderate })^{\S}$ & 0.077 \\
\hline & & iEMG & $52.8 \pm 13.1$ & $52.8 \pm 6.8$ & $0.0 \pm 13.4$ & -0.01 (unclear) & 0.999 \\
\hline & & Mean & $10.7 \pm 3.1$ & $9.6 \pm 2.1$ & $-1.0 \pm 2.4$ & -0.34 (unclear) & 0.338 \\
\hline \multirow{6}{*}{ VM:VL } & \multirow{3}{*}{ ND } & Peak & $85.6 \pm 8.5$ & $97.6 \pm 15.5$ & $11.9 \pm 17.6$ & $1.41{\text { (large })^{\S}}^{8}$ & 0.157 \\
\hline & & iEMG & $87.8 \pm 12.8$ & $101.5 \pm 20.9$ & $13.7 \pm 20.3$ & $0.88(\mathrm{r}$ & 0.124 \\
\hline & & Mean & $84.6 \pm 6.8$ & $98.7 \pm 21.5$ & $14.2 \pm 19.2$ & $2.07{\text { (very large })^{\S}}^{\S}$ & 0.098 \\
\hline & \multirow{3}{*}{ D } & Peak & $94.8 \pm 19.3$ & $98.5 \pm 19.8$ & $3.8 \pm 27.2$ & 0.20 (unclear) & 0.707 \\
\hline & & iEMG & $96.6 \pm 20.9$ & $104.4 \pm 18.3$ & $7.8 \pm 23.1$ & 0.37 (unclear) & 0.340 \\
\hline & & Mean & $100.2 \pm 13.1$ & $110.8 \pm 27.9$ & $10.6 \pm 25.2$ & 0.80 (unclear) & 0.311 \\
\hline \multirow{6}{*}{ RF:BF } & \multirow{3}{*}{ ND } & Peak & $82.6 \pm 24.3$ & $83.3 \pm 9.7$ & $0.7 \pm 30.6$ & 0.0 & 0.955 \\
\hline & & iEMG & $98.3 \pm 34.4$ & $92.9 \pm 18.2$ & $-5.3 \pm 27.1$ & -0.16 (unclear) & 0.620 \\
\hline & & Mean & $120.1 \pm 34.2$ & $106.7 \pm 16.2$ & $-13.4 \pm 19.2$ & $-0.39(\text { small })^{\S}$ & 0.194 \\
\hline & \multirow{3}{*}{ D } & Peak & $92.5 \pm 27.2$ & $106.8 \pm 17.0$ & $14.3 \pm 24.7$ & 0.53 (unclear) & 0.214 \\
\hline & & iEMG & $124.1 \pm 36.9$ & $121.5 \pm 15.1$ & $-2.7 \pm 40.3$ & -0.07 (unclear) & 0.867 \\
\hline & & Mean & $118.3 \pm 43.9$ & $121.8 \pm 27.7$ & $3.6 \pm 37.6$ & 0.09 (unclear) & 0.825 \\
\hline
\end{tabular}




\section{$648 \quad$ Figure captions}

650 Figure 1. Cyclist set-up for data collection with the patella kinesiology-type tape (KTT)

651 applied.

653 Figure 2. Marker placement for 3D motion capture from anterior (left), posterior (middle), 654 and lateral (right) views. Anatomical reference markers were placed bilaterally on the acromial processes, anterior superior iliac spines, posterior superior iliac spines, greater

656 trochanters, medial and lateral femoral epicondyles, medial and lateral malleoli, and $1^{\text {st }}$ and

$6575^{\text {th }}$ metatarsal heads. Tracking markers were placed bilaterally on the heel, mid-foot, and

658 forefoot, and 4-marker rigid clusters were placed on the lateral aspect of the pelvis and

659 bilaterally on the lateral aspects of the thighs and shanks. Anterior superior iliac spine,

660 greater trochanter, femoral epicondyle, malleolus, and $1^{\text {st }}$ metatarsal head markers were removed before the dynamic cycling efforts (red circles).

663 Figure 3. Ratings of comfort levels and perceived change in knee stability and cycling 664 performance with the application of kinesiology-type tape (KTT) compared to no tape (NT) 665 on a 5-point Likert scale. Data presented are the number of cyclists $(n)$ that provided a given 666 rating. Comfort level: 1 , very uncomfortable; 2 , uncomfortable; 3 , no change; 4 , 667 comfortable; 5 , very comfortable. Knee stability: 1 , much less stable; 2 , less stable; 3 , no

668 change; 4, more stable; 5 , much more stable. Performance: 1 , much worse; 2 , worse; 3 , no 669 change; 4 , better; 5 , much better. 